\title{
Pilot Study Examining the Relationship of Childhood Trauma, Perceived Stress, and Medication Use to Serum Kynurenic Acid and Kynurenine Levels in Schizophrenia
}

\author{
Bridget J. Shovestul ${ }^{\mathrm{a}}$, Matthew Glassman ${ }^{\mathrm{a}}$, Laura M. Rowland ${ }^{\mathrm{a}}$, Robert P. McMahon ${ }^{\mathrm{a}}$, Fang \\ Liu $^{a}$, and Deanna L. Kellya \\ aMaryland Psychiatric Research Center, University of Maryland School of Medicine, PO Box \\ 21247, Baltimore, MD 21228
}

\author{
Keywords \\ kynurenic acid; kynurenine; schizophrenia; trauma; stress
}

\section{Dear Editors}

An emerging role of the kynurenine pathway in schizophrenia (SCZ) is supported by post mortem data (Schwarcz et al., 2001) and elevations in serum and cerebral spinal fluid of kynurenic acid (KYNA) and kynurenine in people with SCZ compared to healthy controls (HCs) (Linderholm et al., 2012). However, KYNA levels may be modulated by other factors in humans.

For example, KYNA and kynurenine in SCZ may be related to stress, anxiety, and past trauma. In one study, saliva KYNA increased in patients with SCZ following a stressful task (Chiappelli et al., 2014). Additionally, medications may affect KYNA. The purpose of this preliminary study was to examine the relationships of serum kynurenine and KYNA levels to stress/anxiety, childhood trauma, and medication use in people with SCZ.

In a pilot study, we recruited people with a DSM-IV-TR diagnosis of SCZ or schizoaffective disorder $(N=10)(\mathrm{SCZ})$ and healthy controls $(\mathrm{HC}) \mathrm{s}(N=10)$ having no psychiatric history, between the ages of 18 and 55 years. Five males and females in both groups were involved in a one day visit for blood measurements, clinical ratings, and concomitant medication information. They all went on to participate in a stress paradigm study (Glassman et al., 2015). KYNA and kynurenine were measured by high-performance liquid chromatography

Corresponding Author: Deanna L. Kelly, Pharm.D., BCPP, Maryland Psychiatric Research Center, University of Maryland School of Medicine, PO Box 21247, Baltimore, MD 21228, 410-402-6861 (voice), 410-402-6038 (fax), dkelly @ mprc.umaryland.edu.

Contributors: Bridget Shovestul wrote the first draft of the manuscript and completed the primary statistical analyses. Matthew Glassman helped with the study design, study coordination, and contributed to the final manuscript. Laura Rowland assisted with the study design and contributed to the final manuscript. Robert McMahon served as the study statistician and contributed to the final manuscript. Fang Lu assisted in database organization, management and data set oversight. She also contributed to the final manuscript. Deanna Kelly was principal investigator on the study, conceptualized and oversaw the study implementation and data analyzes. She assisted BS in the final manuscript draft. All authors contributed to and have approved the final manuscript.

Conflict of Interest: All authors have no conflicts of interest to disclose. 
(HPLC) (Pocivavsek et al., 2012). Stress was measured by the Perceived Stress Scale (PSS) (Cohen et al., 1983), and trauma by the Childhood Trauma Questionnaire (CTQ)(Bernstein et al., 1994), which includes a total score and five sub-factors. The State Trait Anxiety Inventory/Visual Analogue Scale (STAI_VAS)(Spielberger, 1983) is comprised of six STAI items and two VAS items. The Mann-Whitney U-test was used for between group differences. Spearman's Rho (R) was used to assess correlations of KYNA and kynurenine with clinical and symptom variables. Difference in the correlation slopes between groups involved the Fisher's Z transformation (z). The Statistical Package for the Social Sciences was used for all analyses.

In our small pilot, participants were similarly matched on smoking, race, and age (mean between 35 and 41 years). There were no differences in serum KYNA ( $40.7 \pm 24.0$ vs. 35.5 $\pm 11.3 \mathrm{nM} ; \mathrm{p}=0.94)$ and kynurenine $(1.53 \pm 0.47$ vs. $1.95 \mu \mathrm{M} \pm 1.5 ; \mathrm{p}=0.47)$ between $\mathrm{SCZ}$ and HCs, respectively. Estimates of effect size using Cohen's d for SCZ versus HCs were d $=+0.28$ for KYNA and -0.89 for kynurenine, demonstrating a trend for higher KYNA levels in SCZ.

Interestingly, the CTQ was significantly correlated with both KYNA (emotional abuse) and kynurenine levels (physical abuse and the total CTQ score) in SCZ, while no relationship existed for HCs (Table 1). Furthermore, the difference in correlation slope between SCZ and $\mathrm{HC}$ for kynurenine levels and the total CTQ score was significant ( $\mathrm{z}=2.03, \mathrm{p}=.042)$, as were the difference in line slopes for kynurenine and physical abuse $(\mathrm{z}=2.06, \mathrm{p}=.039)$ and emotional abuse scores $(\mathrm{z}=2.0, \mathrm{p}=.046)$, suggesting that trauma's association to kynurenine in SCZ plays a role not seen in HCs. The difference in size of correlations between SCZ and HCs for KYNA and the CTQ total score and the 5 domains were not significant ( $p$ >.05).

The PSS and the STAI/VAS scores did not differ between SCZ and HCs. Also in HCs, there were no significant correlations between KYNA or kynurenine levels and measures of anxiety or perceived stress $(\mathrm{p}>0.05)$. However, kynurenine levels and the STAI_tense score were significantly correlated in $\mathrm{SCZ}(\mathrm{R}=.814, \mathrm{p}=.004)$ with a slope difference in this domain noted between SCZ and HC ( $\mathrm{z}=2.45, \mathrm{p}=.014)$.

Within the SCZ group, we found that kynurenine was significantly lower in those taking anti-anxiety medications $(\mathrm{p}=.02)$ and in those taking anticonstipation medications $(\mathrm{p}=.04)$. KYNA levels in SCZ were significantly lower in those taking anticholinergic medications $(\mathrm{p}=.01)$ and anticonstipation medications $(\mathrm{p}=.04)$. Antipsychotic and antidepressant use did not appear to affect KYNA or kynurenine levels.

In conclusion, this is the first study to our knowledge to examine the association of childhood trauma and psychotropic medications to the kynurenine pathway in SCZ. We find that experiencing childhood trauma is associated with increased levels of both kynurenine and KYNA in SCZ, distinct from HC data. Medications for anxiety and those with anticholinergic properties also affect the mean levels of KYNA and kynurenine in SCZ. However, this finding may be due to the presence of anxiety rather than the medications themselves. These findings reflect a potential relationship between kynurenine and anxiety, possibly caused by childhood trauma ( $\mathrm{Li}$ et al., 2016). We have no information on the 
causality of the associations, and it is possible that modulation of kynurenines may be upstream at tryptophan metabolism or other mechanisms, such as cholinergic nicotinic receptors (Wonodi and Schwarcz, 2010). Larger studies are needed to fully address other variables that may modulate KYNA and kynurenine in $\mathrm{SCZ}$ and causation of the effects seen. Increasing knowledge of this potential pathway in $\mathrm{SCZ}$ will aid in personalizing future pharmacologic studies that may target the kynurenine pathway.

\section{Supplementary Material}

Refer to Web version on PubMed Central for supplementary material.

\section{Acknowledgments}

The authors would like to acknowledge Ana Pocivavsek and Francesca Notarangelo for their technical expertise and measurement of kynurenine pathway metabolites.

Funding: Funded in part by Silvio O. Conte Center Grant (Schwarcz P50-MH103222) and Mitsubishi-Tanabe.

\section{References}

Bernstein DP, Fink L, Handelsman L, Foote J, Lovejoy M, Wenzel K, Sapareto E, Ruggiero J. Initial reliability and validity of a new retrospective measure of child abuse and neglect. The American journal of psychiatry. 1994; 151(8):1132-1136. [PubMed: 8037246]

Chiappelli J, Pocivavsek A, Nugent KL, Notarangelo FM, Kochunov P, Rowland LM, Schwarcz R, Hong LE. Stress-induced increase in kynurenic acid as a potential biomarker for patients with schizophrenia and distress intolerance. JAMA psychiatry. 2014; 71(7):761-768. [PubMed: 24806441]

Cohen S, Kamarck T, Mermelstein R. A global measure of perceived stress. Journal of health and social behavior. 1983; 24(4):385-396. [PubMed: 6668417]

Glassman M, Pocivavek A, Notarangelo F, Chiappelli J, Kearns A, Sullivan K, McMahon RP, Liu F, Feldman S, Basham S, Warfel D, Mandelberg A, Rowland L, Kelly DL. Glucocorticoid and inflammatory response to a psychosocial stressor in people with schizophrenia. Schizophrenia bulletin Suppl. 2015; 1:S32.

Li M, D’Arcy C, Meng X. Maltreatment in childhood substantially increases the risk of adult depression and anxiety in prospective cohort studies: systematic review, meta-analysis, and proportional attributable fractions. Psychological medicine. 2016; 46(4):717-730. [PubMed: 26708271]

Linderholm KR, Skogh E, Olsson SK, Dahl ML, Holtze M, Engberg G, Samuelsson M, Erhardt S. Increased levels of kynurenine and kynurenic acid in the CSF of patients with schizophrenia. Schizophrenia bulletin. 2012; 38(3):426-432. [PubMed: 20729465]

Schwarcz R, Rassoulpour A, Wu HQ, Medoff D, Tamminga CA, Roberts RC. Increased cortical kynurenate content in schizophrenia. Biological psychiatry. 2001; 50(7):521-530. [PubMed: 11600105]

Spielberger, CD. Manual for the State-Trait Anxiety Inventory, Form Y. Consulting Psychologists Press; Palo Alto, CA: 1983.

Wonodi I, Schwarcz R. Cortical kynurenine pathway metabolism: a novel target for cognitive enhancement in Schizophrenia. Schizophrenia bulletin. 2010; 36(2):211-218. [PubMed: 20147364] 


\section{Table 1}

Correlations of Childhood Trauma Questionnaire and KYNA/kynurenine

\begin{tabular}{|l|l|l|l|l|}
\hline \multicolumn{3}{|c|}{ Schizophrenia or Schizoaffective disorder } & \multicolumn{2}{c|}{ Healthy Controls } \\
\hline & $\mathrm{KYNA}$ & kynurenine & $\mathrm{KYNA}$ & kynurenine \\
\hline Childhood Trauma Questionnaire Total & $\mathrm{R}=.596, \mathrm{p}=.069$ & $\mathrm{R}=.634, \mathrm{p}=.049^{* * *}$ & $\mathrm{R}=.152, \mathrm{p}=.674$ & $\mathrm{R}=-.324, \mathrm{p}=.361$ \\
\hline Emotional abuse & $\mathrm{R}=.642, \mathrm{p}=.045$ & $\mathrm{R}=.573, \mathrm{p}=.084^{* * *}$ & $\mathrm{R}=.037, \mathrm{p}=.920$ & $\mathrm{R}=-.394, \mathrm{p}=.260$ \\
\hline Physical abuse & $\mathrm{R}=.615, \mathrm{p}=.058$ & $\mathrm{R}=.735, \mathrm{p}=.016^{* * *}$ & $\mathrm{R}=-.177, \mathrm{p}=.625$ & $\mathrm{R}=-.158, \mathrm{p}=.663$ \\
\hline Sexual abuse & $\mathrm{R}=.493, \mathrm{p}=.148$ & $\mathrm{R}=.178, \mathrm{p}=.623$ & $\mathrm{R}=-.017, \mathrm{p}=.962$ & $\mathrm{R}=.495, \mathrm{p}=.146$ \\
\hline Emotional neglect & $\mathrm{R}=.399, \mathrm{p}=.253$ & $\mathrm{R}=.362, \mathrm{p}=.304$ & $\mathrm{R}=.080, \mathrm{p}=.827$ & $\mathrm{R}=-.080, \mathrm{p}=.826$ \\
\hline Physical neglect & $\mathrm{R}=.335, \mathrm{p}=.343$ & $\mathrm{R}=.407, \mathrm{p}=.243$ & $\mathrm{R}=.450, \mathrm{p}=.192$ & $\mathrm{R}=-.452, \mathrm{p}=.190$ \\
\hline
\end{tabular}

depicts a significant difference in correlation size from the HC group when looking at the relationship between the same variables using Fisher's $\mathrm{Z}$ transformation 